\title{
Covid-19 Vaccination; Where Do We Stand?
}

\author{
Naeemullah
}

This editorial may be cited as: Naeemullah.

Covid-19 Vaccination; Where Do We Stand? J Saidu Med Coll Swat 2021;11(2): 61-62

COVID-19 is a global pandemic ${ }^{1}$. It has affected almost all countries, territories and communities worldwide and is a global health tragedy of the century after World War II'.

Appearance of COVID-19 caused by the SARS COVID-19 virus there has been sudden increase of vaccine development around the globe. Currently vaccines have been commonly well thought-out as part of exit strategy to go back to the normal life and attain herd immunity ${ }^{3}$. Around the world many vaccines are in pre-clinical development and some entered in clinical trials including approaches which have not been previously been licensed for human vaccines. Several countries such as Israel (92 doses per 100 people) and United Arab Emirates are making progress in immunizing their citizens ${ }^{4}$.

Clinical trials of four vaccines are showing remarkable promise with $90 \%$ efficacy but there is still a large amount of vaccines hesitancy exists among community around the world. Vaccines hesitancy poses dangers to both the individuals and their community exposure to a contagious diseases puts the person to and they are far more likely to spread to others if they won't get vaccinated $^{5}$. By some estimates about $30 \%$ Americans will refuse the COVID-19 Vaccine. The Vaccination efforts could soon move from supply and logistics to individual's reluctance to be vaccinated $^{6}$.

Pakistan formally launched the COVID-19 vaccination drive on Feb $3^{\text {rd }} 2021$ but poor response has been witness by front line health care workers in Pakistan especially in Khyber Pakhtunkhwa(KP) although KP is the highest with a crude fatality rate of $4.9 \%$ and many health workers lost their lives in the battle against corona virus $^{7}$. The practical strategies would be to educate masses by creating more webinars and seminars to explain what's known and what's not.

Correspondence: Dr. Naeemullah

Department of Community Medicine

Saidu Medical College, Saidu Sharif, Swat

E-mail: pediatrician777@hotmail.com
Vaccine and rapid diagnostic are the single COVID-19 exit approach ${ }^{8}$. In Pakistan the vaccination refusal rate is extremely high, and is associated with lack of ability to read or write, joblessness and low socio economic status ${ }^{9}$.

Vaccine hesitancy remains a substantial challenge in Pakistan because of different conspiracy theories, which are attributed to the failure of complete eradication of polio from the country. The reasons are poor or low quality of vaccine, alleged dosing recommendations, some religious prohibitions, and different rumors regarding the presence of vigorous virus in the vaccines are few principal claims obstructing the anti-polio campaign in the country. In Pakistan unfortunately, a conspiracy theory against COVID-19 vaccine is currently being spread ${ }^{10}$.

In Pakistan vaccine hesitancy poses serious challenges for achieving coverage for population immunity. It is necessary to achieve high COVID19 vaccination acceptance rates in health care workers and community ${ }^{11}$. The vaccine should be equitably and justly distributed, particularly targeting individuals at highest risk for complications and disease transmission to others if initial vaccine supply does not meet demand ${ }^{12}$.

Ensuring the accessibility, availability and affordability of vaccines of life-saving COVID-19 vaccines is one of the three recommendations of the Global Vaccine Action Plan to close the immunization gap. In Pakistan health care workers and older citizens are taking vaccines in first phase and it is observed that they reluctant to take vaccines like polio and other vaccines, if health care workers are reluctant to vaccines then how general population will trust and take it. So the government needs to ensure accessibility, availability and affordability of vaccines to all the citizens of the country and also to ensure proper advocacy on mass scale for COVID-19 vaccine acceptance. 


\section{REFERENCES}

1. Jebril N. World Health Organization declared a pandemic public health menace: Asystematic review of the coronavirus disease 2019 "COVID-19", up to 26th March 2020. Available SSRN 3566298. 2020;

2. Acter T, Uddin N, Das J, Akhter A, ChoudhuryTR, Kim $S$. Evolution of severe acute respiratory syndrome coronavirus 2 (SARS-CoV-2) as coronavirus disease 2019 (COVID-19) pandemic: A global health emergency. Sci Total Environ. 2020;1389-96.

3. Tregoning JS, Brown ES, Cheeseman HM, Flight KE, Higham SL, Lemm N, et al. Vaccines for COVID-19. Clin Exp Immunol. 2020;202(2):162-92.

4. Analytica O. Risks and opportunities 2021: COVID19. Emerald Expert Briefings. (oxan-ga).

5. Bhopal S, Nielsen M. Vaccine hesitancy in low-and middle-income countries: Potential implications for the COVID-19 response. Arch Dis Child. 2021;106(2):11-34.

6. Makvandi M, Wallis LD, West CN, De Angelis HE, VanWinkle Z, Halkjaer-Knudsen V, et al. Modeling efficient and equitable distribution of COVID-19 vaccines. Sandia National Lab.(SNL-NM), Albuquerque, NM (United States); 2020.

7. Noreen N, Dil S, Niazi S, Naveed I, Khan N, Khan F, et al. COVID-19 pandemic \& Pakistan; limitations and gaps. Glob Biosecurity. 2020;1(4).

8. Jamaludin S, Azmir NA, Ayob AFM, Zainal N. COVID19 exit strategy: Transitioning towards a new normalReview article. Ann Med Surg. 2020;

9. Khattak FA, Rehman K, Shahzad M, Arif N, Ullah N, Kibria Z, et al. Prevalence of Parental refusal rate and its associated factors in routine immunization by using WHO Vaccine Hesitancy tool:A Cross sectional study at district Bannu, KP, Pakistan. Int J Infect Dis. 2021;104:117-24.

10. Khan YH, Mallhi TH, Alotaibi NH, Alzarea Al, Alanazi AS, Tanveer N, et al. Threat of COVID-19 vaccine hesitancy in Pakistan: the need for measures to neutralize misleading narratives. Am J Trop Med Hyg. 2020;103(2):60-34.

11. Boyle P, Langman MJS. ABC of colorectal cancer: Epidemiology. Bmj. 2000;321(Suppl S6).

12. Saied SM, Saied EM, Kabbash IA, Abdo SAE. Vaccine hesitancy: Beliefs and barriers associated with COVID-19 vaccination among Egyptian medical students. J Med Virol. 2021; 